\title{
Systematic Ultimate Bound Computation for Sampled-data Systems with Quantization
}

\author{
Hernan Haimovich ${ }^{a, 1}$ Ernesto Kofman ${ }^{a}$ María M. Seron ${ }^{b}$ \\ ${ }^{a}$ CONICET; Laboratorio de Sistemas Dinámicos y Procesamiento de la Información, Facultad de Cs. Exactas, Ingeniería y Agrimensura, \\ Universidad Nacional de Rosario, Riobamba 245bis, 2000 Rosario, Argentina \\ ${ }^{\mathrm{b}}$ Centre for Complex Dynamic Systems and Control (CDSC), The University of Newcastle, Callaghan, NSW 2308, Australia
}

\begin{abstract}
We present a novel systematic method to obtain componentwise ultimate bounds in perturbed sampled-data systems, especially when the perturbations arise due to quantization. The proposed method exploits the system geometry as well as the perturbation structure, and takes intersample behavior into account. The main features of the method are its systematic nature, whereby it can be readily computer coded, without requiring adjustment of parameters for its application, and its suitability for dealing with highly structured perturbation schemes, whereby the information on the perturbation structure is directly taken into account. The latter feature distinguishes the method from other approaches that require a bound on the norm of the perturbation and thus disregard information on the perturbation structure. We apply the method to a numerical example taken from the literature to illustrate its simplicity and potential.
\end{abstract}

Key words: Ultimate bounds, quantized sampled-data systems, bounded perturbation, componentwise bounds, non Lyapunov analysis.

\section{Introduction}

This paper deals with the computation of guaranteed ultimate bounds for sampled-data systems that involve quantization. Quantization in control systems has been studied for nearly 50 years (Bertram, 1958). The usual approach to analyzing the effects of quantization on a digital control system has been to regard a quantized variable as a perturbed copy of the unquantized variable, where the perturbation is modelled as white noise (Williamson, 1991). This approach leads to statistical analysis of the effects of quantization and has proven very useful in control applications. However, statistical description of the quantization error may lead to unnecessary difficulties if only a guaranteed ultimate bound is sought. Methods that employ a deterministic framework also exist (see e.g., Yakowitz and Parker, 1973; Green and Turner, 1988; Miller et al., 1989). These latter methods also regard a quantized variable as a perturbed copy of the unquantized variable.

More recently, sampled-data systems involving quantization have attracted renewed interest due to the success of networked control systems (see Antsaklis and Baillieul, eds., 2004, and the references therein). Many works in this emerg-

\footnotetext{
${ }^{1}$ Corresponding author. Ph: +543414808543 ext.106, Fax: +54 341 4802654, email: haimodfceia.unr.edu.ar
}

ing area explicitly consider quantization, without regarding a quantized variable as a perturbed copy (e.g., Wong and Brockett, 1999; Elia and Mitter, 2001; Ishii and Francis, 2003; Ishii et al., 2004; Ishii and Başar, 2005). Some of these works ensure the asymptotic stability of the resulting closedloop system by, e.g., employing quantizers with increasingly higher precision as the state approaches the origin (as with logarithmic quantizers in Elia and Mitter, 2001). Our focus in this paper is on cases where asymptotic stability cannot be ensured and thus computing an ultimate bound on the system trajectories is of interest. We will approach the problem by regarding a quantized variable as a perturbed copy of the corresponding unquantized variable, as is the case in the works mentioned in the previous paragraph. However, we will bound the perturbations introduced by the quantizers in a novel (and tighter) form and will apply our results to an example taken from Ishii et al. (2004).

For purely continuous- or discrete-time perturbed systems, i.e., in the absence of sampling and hold devices in the control loop, a typical approach to estimate ultimate bounds is via level sets of suitable Lyapunov functions (Khalil, 2002). This approach has the advantage of being very general and powerful and the disadvantage of requiring the obtention of a suitable Lyapunov function. For linear systems, however, quadratic Lyapunov functions can be easily computed. A closely related approach to estimate ultimate bounds is 
via the input-to-state stability (ISS) framework for systems with disturbances (Sontag and Wang, 1995; Jiang and Wang, 2001). In the context of quantized sampled-data systems, this framework could be employed by considering the quantization effects as disturbances, obtaining a discrete-time ISS characterization for the sampled-data system at the sampling instants (Huang et al., 2005), and then using the results of Nešić et al. (1999) to derive an ISS characterization for the sampled-data system. However, this methodology is not directly applicable if the disturbance bounds may depend on the system state (as is the case when considering logarithmic quantizers). In addition, both the Lyapunov function and ISS methods require a bound on the norm of the perturbation and may lead to conservative bounds if important information on the structure of the perturbation is lost when bounding its norm.

A method to estimate ultimate bounds that does not require the selection of a Lyapunov function, nor bounding the norm of the perturbation, was introduced in Kofman (2005) for continuous-time linear time-invariant (LTI) systems and extended in Kofman et al. (2007). These works presented a new framework to obtain closed-form ultimate bound formulae based on the use of componentwise perturbation bounds and componentwise analysis of the system. As compared with the bounds obtained by means of the standard quadratic-Lyapunov-function approach, the examples in Kofman (2005) and Kofman et al. (2007) show that this componentwise framework may in some cases provide much tighter bounds. This componentwise framework has been developed for purely continuous- and discrete-time systems and cannot be directly applied to sampled-data systems. Moreover, when the perturbation bound depends on the system state, Kofman et al. (2007) provides ultimate bounds that are only locally valid.

In this context, the main contribution of the current paper is a systematic method to obtain componentwise ultimate bounds in perturbed sampled-data systems, which provides bounds that are globally valid, utilizes a novel form for the perturbation bound, and is especially suited to perturbations introduced by quantization. We follow ideas similar to those used in Kofman (2005) and Kofman et al. (2007), and derive new results that can be directly applied to perturbed sampleddata systems to obtain global ultimate bounds. These bounds take intersample behavior into account and can be calculated in a systematic way, i.e., they do not require adjustment of parameters or selection of suitable norms or Lyapunov functions.

Notation. For a matrix $M$ with (real or complex) entries $M_{i, j}$, let $|M|$ and $\mathbb{R e}(M)$ denote the elementwise magnitude and real part, respectively; $M \leq N$ and $M<N$ the sets of componentwise inequalities $M_{i, j} \leq N_{i, j}$ and $M_{i, j}<$ $N_{i, j}$, respectively, and similarly for $M \geq N$ and $M>N$; $\mathbb{R}_{+}, \mathbb{R}_{+, 0}$ the sets of positive and nonnegative real numbers, respectively; and $\rho(M)$ the spectral radius of matrix $M$.

\section{The quantized sampled-data system}

We consider the sampled-data system depicted in Figure 1, described by

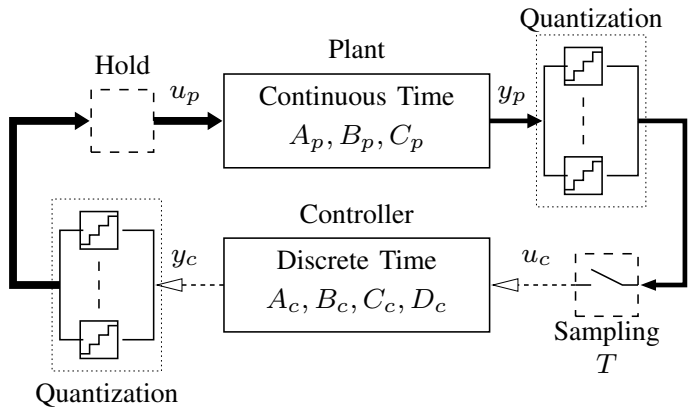

Fig. 1. Quantized sampled-data system.

$$
\begin{aligned}
& \dot{x}_{p}(t)=A_{p} x_{p}(t)+B_{p} u_{p}(t), \quad y_{p}(t)=C_{p} x_{p}(t), \\
& x_{c}(k+1)=A_{c} x_{c}(k)+B_{c} u_{c}(k), \\
& y_{c}(k)=C_{c} x_{c}(k)+D_{c} u_{c}(k), \\
& u_{c}(k)=y_{p}\left(t_{k}\right)+\Delta y_{p}\left(t_{k}\right), \\
& u_{p}(t)=y_{c}(k)+\Delta y_{c}(k), \quad t_{k} \leq t<t_{k}+\mathrm{T},
\end{aligned}
$$

where $x_{p}(t) \in \mathbb{R}^{\mathrm{N}_{p}}, u_{p}(t) \in \mathbb{R}^{\mathrm{M}}$ and $y_{p}(t) \in \mathbb{R}^{\mathrm{P}}$ are the continuous-time plant state, input and output, respectively, $x_{c}(k) \in \mathbb{R}^{\mathrm{N}_{c}}, u_{c}(k) \in \mathbb{R}^{\mathrm{P}}$ and $y_{c}(k) \in \mathbb{R}^{\mathrm{M}}$ are the discretetime controller state, input and output, respectively, $\Delta y_{p}$ and $\Delta y_{c}$ are the perturbations introduced by the quantizers at the plant and controller outputs, respectively, T is the sampling period, and $t_{k}=k \mathrm{~T}$ for $k=0,1, \ldots$ Each individual connection signal between plant and controller has an independent quantizer, each of which can have different features and can be of uniform, logarithmic or semipractical logarithmic type.

\section{Discrete-time model.}

The model of the system at the sampling instants is

$$
\begin{aligned}
& x_{k+1}=A_{d} x_{k}+B_{d} \Delta y_{k}, \quad \text { where } \\
& x_{k} \triangleq\left[\begin{array}{c}
x_{p}\left(t_{k}\right) \\
x_{c}(k)
\end{array}\right] \in \mathbb{R}^{n}, \quad \Delta y_{k} \triangleq\left[\begin{array}{c}
\Delta y_{p}\left(t_{k}\right) \\
\Delta y_{c}(k)
\end{array}\right] \in \mathbb{R}^{\mathrm{S}}, \\
& n \triangleq \mathrm{N}_{p}+\mathrm{N}_{c}, \mathrm{~S} \triangleq \mathrm{P}+\mathrm{M} .
\end{aligned}
$$

and $A_{d}$ and $B_{d}$ can be directly obtained from (1) as

$$
\begin{array}{rlrl}
A_{d} & =\left[\begin{array}{cc}
A_{11} & A_{12} \\
B_{c} C_{p} & A_{c}
\end{array}\right], & & B_{d}=\left[\begin{array}{cc}
B_{11} & B_{12} \\
B_{c} & 0
\end{array}\right], \\
A_{11} & \triangleq e^{A_{p} \mathrm{~T}}+\Psi(\mathrm{T}) B_{p} D_{c} C_{p}, & A_{12} \triangleq \Psi(\mathrm{T}) B_{p} C_{c}, \\
B_{11} & \triangleq \Psi(\mathrm{T}) B_{p} D_{c}, & B_{12} \triangleq \Psi(\mathrm{T}) B_{p}, \\
\Psi(t) & \triangleq \int_{0}^{t} e^{A_{p} \tau} d \tau . &
\end{array}
$$


In the remainder of this section, we derive componentwise bounds on the perturbation vector $\Delta y_{k}$ [see (3)], according to the type and features of the quantizers employed for each signal.

\section{Individual quantizer perturbations.}

We regard a scalar quantized variable, $q(s)$, as a perturbed copy of the unquantized variable: $q(s)=s+\Delta s$. We next derive bounds on $\Delta s$ when $q$ is uniform, logarithmic or semipractical logarithmic. A uniform quantizer has uniformly spaced levels. In this case, the quantizer perturbation $\Delta s$ can be bounded by $|\Delta s| \leq u^{\circ} \triangleq \alpha / 2$, where $\alpha$ is the quantization step. A logarithmic quantizer has levels in a set $U \subset \mathbb{R}$ defined as

$$
U=\left\{ \pm \rho^{-i} u^{\circ}, i=0, \pm 1, \pm 2, \ldots\right\} \cup\{0\},
$$

where $0<\rho<1$ and $u^{\circ}>0$. The quantizer perturbation satisfies $|\Delta s| \leq \delta|s|$, where $\delta=\frac{1-\rho}{1+\rho}$. Practical logarithmic quantizers arise from truncating a logarithmic quantizer so that the resulting quantizer has only a finite number of levels. We consider a semipractical quantizer in the sense that it is truncated only towards the origin, i.e., it has values in the set $U=\left\{ \pm \rho^{-i} u^{\circ}, i=0,1,2, \ldots\right\} \cup\{0\}$ [cf. (9)], where $0<\rho<1$ and $u^{\circ}>0$. In this case,

$$
|\Delta s| \leq \max \left\{\delta|s|, \frac{u^{\circ}}{1+\delta}\right\} .
$$

Note that (10) encompasses the three types of quantizer perturbations considered, i.e., the other perturbation bounds can be obtained from (10) by adjusting $\delta$ and $u^{\circ}$.

Remark 1 Without considering overflow, the perturbation bound (10) can accommodate the effects of analog-to-digital conversion using fixed-or floating-point representation. The form of the perturbation bound (10) is one key difference in our approach, since it can be made tighter than the usual bound for the perturbation introduced when considering floating-point representations, of the form $|\Delta s| \leq \delta|s|+\gamma$ (see e.g., Miller et al., 1988).

\section{Quantizer perturbations in vector form.}

Using the single-quantizer-perturbation bound (10) and defining

$$
\begin{aligned}
& \Gamma_{p} \triangleq \operatorname{diag}\left(\delta_{1}, \ldots, \delta_{\mathrm{P}}\right), \quad \Gamma_{c} \triangleq \operatorname{diag}\left(\delta_{\mathrm{P}+1}, \ldots, \delta_{\mathrm{S}}\right), \\
& \theta_{p} \triangleq\left[\begin{array}{lll}
\frac{u_{1}^{\circ}}{1+\delta_{1}} & \cdots & \frac{u_{\mathrm{P}}^{\circ}}{1+\delta_{\mathrm{P}}}
\end{array}\right]^{\prime} \quad \theta_{c} \triangleq\left[\begin{array}{ccc}
\frac{u_{\mathrm{P}+1}^{\circ}}{1+\delta_{\mathrm{p}+1}} & \cdots & \frac{u_{\mathrm{s}}^{\circ}}{1+\delta_{\mathrm{s}}}
\end{array}\right]^{\prime}
\end{aligned}
$$

where $\delta_{i}$ and $u_{i}^{\circ}$ correspond to the quantizer at the $i$-th plant output for $i=1, \ldots, \mathrm{P}$, and at the $(i-\mathrm{P})$-th controller output for $i=\mathrm{P}+1, \ldots, \mathrm{s}$, it follows that

$$
\begin{aligned}
\left|\Delta y_{p}\left(t_{k}\right)\right| & \leq \max \left\{\Gamma_{p}\left|y_{p}\left(t_{k}\right)\right|, \theta_{p}\right\}, \\
\left|\Delta y_{c}(k)\right| & \leq \max \left\{\Gamma_{c}\left|y_{c}(k)\right|, \theta_{c}\right\},
\end{aligned}
$$

where the maximum is taken componentwise. In (13)-(14), $y_{p}\left(t_{k}\right)$ and $y_{c}(k)$ satisfy, from (1),

$$
\begin{aligned}
y_{p}\left(t_{k}\right) & =C_{p} x_{p}\left(t_{k}\right), \\
y_{c}(k) & =C_{c} x_{c}(k)+D_{c} C_{p} x_{p}\left(t_{k}\right)+D_{c} \Delta y_{p}\left(t_{k}\right) .
\end{aligned}
$$

In Section 3, we will need bounds on $\Delta y_{p}\left(t_{k}\right)$ and $\Delta y_{c}(k)$ in terms of a transformed version of the state. Therefore, let $V \in \mathbb{C}^{n \times n}$ denote an arbitrary invertible matrix and consider the transformation $x_{k}=V z_{k}$, where $V$ is partitioned according to $x_{k}$ in (3) as

$$
V=\left[\begin{array}{ll}
V_{p}^{\prime} & V_{c}^{\prime}
\end{array}\right]^{\prime} \text {, where } V_{p} \in \mathbb{C}^{\mathrm{N}_{p} \times n} \text { and } V_{c} \in \mathbb{C}^{\mathrm{N}_{c} \times n} \text {. }
$$

Operating on (13)-(16), and using (17) yields ${ }^{2}$

$$
\begin{aligned}
\left|\Delta y_{p}\left(t_{k}\right)\right| & \leq Y\left(\left|z_{k}\right|\right) \triangleq \max \left\{\Theta_{p}\left|z_{k}\right|, \theta_{p}\right\}, \\
\left|\Delta y_{c}(k)\right| & \leq \max \left\{\Theta_{c}\left|z_{k}\right|+\Theta_{s} Y\left(\left|z_{k}\right|\right), \theta_{c}\right\}, \\
\Theta_{p} & \triangleq \Gamma_{p}\left|C_{p} V_{p}\right|, \quad \Theta_{s} \triangleq \Gamma_{c}\left|D_{c}\right|, \\
\Theta_{c} & \triangleq \Gamma_{c}\left|C_{c} V_{c}+D_{c} C_{p} V_{p}\right| .
\end{aligned}
$$

\section{Ultimate bounds for the sampled-data system}

\subsection{Discrete-time state trajectory bound}

The following theorem provides componentwise bounds on the state of the perturbed discrete-time system (2)-(3) when the perturbation $\Delta y_{k}$ is bounded as in (18)-(19). In Section 3.2, we will derive componentwise bounds on the plant states that, in addition, take account of intersample behavior.

Theorem 2 Consider system (2)-(3) and express $A_{d}$ in Jordan canonical form as $A_{d}=V \Lambda V^{-1}$. Let $\Delta y_{k}$ be bounded as in (18)-(19) for all $k \geq 0$, where $z_{k}=V^{-1} x_{k}, \Theta_{p} \in$ $\mathbb{R}_{+, 0}^{\mathrm{P} \times n}, \Theta_{c} \in \mathbb{R}_{+, 0}^{\mathrm{M} \times n}, \theta_{p} \in \mathbb{R}_{+, 0}^{\mathrm{P}}, \theta_{c} \in \mathbb{R}_{+, 0}^{\mathrm{M}}$, and $\Theta_{s} \in \mathbb{R}_{+, 0}^{\mathrm{M} \times \mathrm{P}}$. Define

$$
\begin{aligned}
M & \triangleq|\Lambda|+\left|V^{-1} B_{d}\right| \Theta, \\
\Theta & \triangleq\left[\begin{array}{c}
\Theta_{p} \\
\Theta_{c}+\Theta_{s} \Theta_{p}
\end{array}\right], \quad \theta \triangleq\left[\begin{array}{c}
\theta_{p} \\
\theta_{c}+\Theta_{s} \theta_{p}
\end{array}\right],
\end{aligned}
$$

and suppose that $\rho(M)<1$. Then, $\rho(|\Lambda|)<1$. Define

$$
\beta \triangleq(\mathrm{I}-M)^{-1}\left|V^{-1} B_{d}\right| \theta, \quad \gamma \triangleq(\mathrm{I}-|\Lambda|)^{-1}\left|V^{-1} B_{d}\right|\left[\theta_{p}^{\prime} \theta_{c}^{\prime}\right]^{\prime} .
$$

Then $\beta \geq 0$ and $\gamma \geq 0$. Consider the map $Y(\cdot)$ defined in (18), the map $T: \mathbb{R}_{+, 0}^{n} \rightarrow \mathbb{R}_{+, 0}^{n}$ defined by

$$
T(w)=|\Lambda| w+\left|V^{-1} B_{d}\right|\left[\begin{array}{c}
\max \left\{\Theta_{p} w, \theta_{p}\right\} \\
\max \left\{\Theta_{c} w+\Theta_{s} Y(w), \theta_{c}\right\}
\end{array}\right],
$$

and the sequence $\left\{b_{r}\right\}_{r=0}^{\infty}$ defined by $b_{0} \triangleq \beta$ and $b_{r} \triangleq$ $T^{r}(\beta)$ for $r=1,2, \ldots$ Then,

\footnotetext{
${ }^{2}$ For an introduction to the properties of matrices with nonnegative entries, see, e.g., Horn and Johnson $(1985, \S 8.1)$.
} 
1. $0 \leq b_{r} \leq b_{r-1}$ for $r=1,2, \ldots$ and $b_{\infty} \triangleq \lim _{r \rightarrow \infty} T^{r}(\beta)$ exists and satisfies $0 \leq \gamma \leq b_{\infty} \leq \beta$.

2. Fix any $x_{0} \in \mathbb{R}^{n}$ and define the sequence $\left\{\bar{\beta}_{r}\right\}_{r=0}^{\infty}$ by $\bar{\beta}_{r}=\max \left\{\left|V^{-1} x_{0}\right|, b_{r}\right\}$. Then, for all $k \geq 0$ and all $r \geq 0$,

a) $\left|V^{-1} x_{k}\right| \leq b_{r+k}+M^{k}\left(\bar{\beta}_{r}-b_{r}\right)$.

b) $\left|x_{k}\right| \leq|V| b_{r+k}+|V| M^{k}\left(\bar{\beta}_{r}-b_{r}\right)$.

3. For any $x_{0} \in \mathbb{R}^{n}$, $\lim \sup _{k \rightarrow \infty}\left|V^{-1} x_{k}\right| \leq b_{\infty}$ and $\lim \sup _{k \rightarrow \infty}\left|x_{k}\right| \leq|V| b_{\infty}$, where the limsup operates componentwise.

PROOF. Note that $|\Lambda|,\left|V^{-1} B_{d}\right|, \Theta$ and $M$ all have nonnegative entries. Then, from (22), it follows that $\rho(M) \geq$ $\boldsymbol{\rho}(|\Lambda|)+\boldsymbol{\rho}\left(\left|V^{-1} B_{d}\right| \Theta\right)$ (see, e.g., Horn and Johnson, 1985, $\S 8.1$, and by assumption, $\rho(M)<1$. Since the spectral radius is a nonnegative quantity, it follows that $\rho(|\Lambda|)<1$. Note then that $\mathrm{I}-M$ and $\mathrm{I}-|\Lambda|$ are invertible and $\beta$ and $\gamma$ in (24), are well defined. Define the map $T_{M}: \mathbb{R}_{+, 0}^{n} \rightarrow \mathbb{R}_{+, 0}^{n}$ as

$$
T_{M}(w)=M w+\left|V^{-1} B_{d}\right| \theta .
$$

Note that, for any $w \in \mathbb{R}_{+, 0}^{n}, T_{M}(w) \geq 0$ and hence $T_{M}^{r}(w) \geq 0$ for all $r \geq 0$. Since $\rho(M)<1$, then $\lim _{r \rightarrow \infty} T_{M}^{r}(w)=\beta \geq 0$ for all $w \in \mathbb{R}_{+, 0}^{n}$. The proof that $\gamma \geq 0$ follows similarly by considering the map defined by $T_{|\Lambda|}(w)=|\Lambda| w+\left|V^{-1} B_{d}\right|\left[\theta_{p}^{\prime} \theta_{c}^{\prime}\right]^{\prime}$.

1. Since the matrices $|\Lambda|,\left|V^{-1} B_{d}\right|, \Theta_{p}, \Theta_{c}$, and $\Theta_{s}$, and the vectors $\theta_{p}, \theta_{c}$ all have nonnegative entries, it follows that

$$
\begin{gathered}
w_{1} \leq w_{2} \Rightarrow T\left(w_{1}\right) \leq T\left(w_{2}\right), \quad \text { and } \\
T(w) \leq|\Lambda| w+\left|V^{-1} B_{d}\right|(\Theta w+\theta)=T_{M}(w),
\end{gathered}
$$

for all $w_{1}, w_{2}, w \in \mathbb{R}_{+, 0}^{n}$. From (24) and (26), note that $\beta=T_{M}(\beta)$, whence, using (28), $b_{1}=T(\beta) \leq \beta=b_{0}$. Applying (27) iteratively to the latter inequality yields $b_{r}=$ $T^{r}(\beta) \leq T^{r-1}(\beta)=b_{r-1}$. The sequence $\left\{b_{r}\right\}_{r=0}^{\infty}$ is thus componentwise nonincreasing. Moreover, this sequence is componentwise lower bounded by zero since $b_{r}=T^{r}(\beta)$, $\beta \geq 0$ and $T: \mathbb{R}_{+, 0}^{n} \rightarrow \mathbb{R}_{+, 0}^{n}$. Hence, $\left\{b_{r}\right\}_{r=0}^{\infty}$ converges to some point $b_{\infty} \stackrel{=}{=} \lim _{r \rightarrow \infty} b_{r}$ satisfying $0 \leq b_{\infty} \leq \beta$. From (25) and since $T\left(b_{\infty}\right)=b_{\infty}$, note that $b_{\infty} \geq|\Lambda| b_{\infty}+$ $\left|V^{-1} B_{d}\right|\left[\theta_{p}^{\prime} \theta_{c}^{\prime}\right]^{\prime}$. Then, $(\mathrm{I}-|\Lambda|) b_{\infty} \geq\left|V^{-1} B_{d}\right|\left[\theta_{p}^{\prime} \theta_{c}^{\prime}\right]^{\prime}$ and therefore, from (24), $b_{\infty} \geq \gamma$, since $|\Lambda|$ is in Jordan form, has nonnegative entries, and $\rho(|\Lambda|)<1$.

2. Let $x_{k}=V z_{k}$. Taking magnitudes and using (2)-(3), (18)-(19), and (25) yields

$$
\left|z_{k+1}\right| \leq|\Lambda|\left|z_{k}\right|+\left|V^{-1} B_{d}\right|\left|\Delta y_{k}\right| \leq T\left(\left|z_{k}\right|\right)
$$

Using (27), (29), and noting that $\left|V^{-1} x_{0}\right|=\left|z_{0}\right| \leq \bar{\beta}_{r}$ for all $r \geq 0$, then

$$
\left|z_{k}\right| \leq T^{k}\left(\left|z_{0}\right|\right) \leq T^{k}\left(\bar{\beta}_{r}\right), \quad \forall k, r \geq 0
$$

Claim: Let $w, y \in \mathbb{R}_{+, 0}^{n}$ and suppose that $w \geq y \geq 0$. Then, $0 \leq T(w)-T(y) \leq M(w-y)$. For a proof of this claim, see Haimovich $(2006, \S 6)$.

Since $\bar{\beta}_{r} \geq b_{r} \geq 0$, by the claim then $0 \leq T\left(\bar{\beta}_{r}\right)-$ $T\left(b_{r}\right) \leq \bar{M}\left(\bar{\beta}_{r}-b_{r}\right)$. Then, $T\left(\bar{\beta}_{r}\right) \geq T\left(b_{r}\right) \geq 0$ and using the claim again yields $T^{2}\left(\bar{\beta}_{r}\right)-\bar{T}^{2}\left(b_{r}\right) \leq \bar{M}\left(T\left(\bar{\beta}_{r}\right)-\right.$ $\left.T\left(b_{r}\right)\right) \leq M^{2}\left(\bar{\beta}_{r}-b_{r}\right)$. Iterating this procedure yields $T^{k}\left(\bar{\beta}_{r}\right)-T^{k}\left(b_{r}\right) \leq M^{k}\left(\bar{\beta}_{r}-b_{r}\right)$ and hence, operating and combining with (30) yields

$$
\left|z_{k}\right| \leq T^{k}\left(\bar{\beta}_{r}\right) \leq M^{k}\left(\bar{\beta}_{r}-b_{r}\right)+T^{k}\left(b_{r}\right) .
$$

Part 2 a) then follows straightforwardly since $\left|z_{k}\right|=$ $\left|V^{-1} x_{k}\right|$ and $T^{k}\left(b_{r}\right)=b_{r+k}$. To prove $\left.2 \mathrm{~b}\right)$ note that $\left|x_{k}\right| \leq|V|\left|V^{-1} x_{k}\right|$ and use 2 a).

3. From 2 a), we can write

$$
\begin{gathered}
\limsup _{k \rightarrow \infty}\left|V^{-1} x_{k}\right| \leq \limsup _{k \rightarrow \infty}\left[M^{k}\left(\bar{\beta}_{r}-b_{r}\right)+b_{r+k}\right] \\
\leq \limsup _{k \rightarrow \infty} M^{k}\left(\bar{\beta}_{r}-b_{r}\right)+\limsup _{k \rightarrow \infty} b_{r+k} .
\end{gathered}
$$

Since $\lim _{k \rightarrow \infty} M^{k}\left(\bar{\beta}_{r}-b_{r}\right)=0$ because $\boldsymbol{\rho}(M)<1$, and $\lim _{k \rightarrow \infty} b_{r+k}=b_{\infty}$ by part 1 , then $\lim \sup _{k \rightarrow \infty}\left|V^{-1} x_{k}\right| \leq$ $b_{\infty}$, establishing $3 \mathrm{a}$ ). To prove $3 \mathrm{~b}$ ), use $\left|x_{k}\right| \leq|V|\left|V^{-1} x_{k}\right|$ in 3 a).

Theorem 2 provides a systematic method to compute componentwise bounds on the state trajectories of a discretetime system of the form (2)-(3) where the perturbation $\Delta y_{k}$ is bounded as in (18)-(19). In particular, if the matrices in (18)-(19) have the form (20)-(21), then Theorem 2 provides bounds for the quantized sampled-data system structure of Figure 1 at the sampling instants. In addition, Theorem 2 part 3 directly provides global ultimate bounds on the system state.

Remark 3 Combining parts 1 and 3 of Theorem 2, note that $\lim \sup _{k \rightarrow \infty}\left|V^{-1} x_{k}\right| \leq b_{\infty} \leq b_{r}$, for any $r \geq 0$. Therefore, $b_{r}$ can also be used to compute an ultimate bound for the system. Such a bound, though being more conservative than that corresponding to $b_{\infty}$, has the advantage of requiring only $r$ iterations of the map $T$ defined in (25).

Remark 4 If $\left|V^{-1} x_{0}\right| \leq b_{r}$ for some $r \geq 0$, then Theorem 2 parts 2 and 1 yield $\left|V^{-1} x_{k}\right| \leq b_{r+k} \leq b_{r}$, since in this case $\bar{\beta}_{r}=b_{r}$. Therefore, each of the regions $\left\{x \in \mathbb{R}^{n}:\left|V^{-1} x\right| \leq\right.$ $\left.b_{r}\right\}, 0 \leq r \leq \infty$, is invariant under the system dynamics.

Remark 5 Theorem 2 requires that $M$ in (22) have all its eigenvalues in the open unit disc $(\rho(M)<1)$. Note then that $\boldsymbol{\rho}(M)<1$ is a sufficient condition for global practical stability of the perturbed discrete-time system considered, i.e., for its trajectories to be ultimately bounded from any initial condition. Moreover, since $\rho(M)<1$ implies that 
$\boldsymbol{\rho}(|\Lambda|)=\boldsymbol{\rho}(\Lambda)=\boldsymbol{\rho}\left(A_{d}\right)<1$, then a necessary condition for application of Theorem 2 is that the unperturbed discretetime system $x_{k+1}=A_{d} x_{k}$ be stable.

\subsection{Ultimate bound with intersample}

The following theorem combines the ultimate bound derived in Theorem 2 part 3 with a bound on the variation of the plant states between sampling instants, providing a componentwise ultimate bound on the plant states of the sampleddata system considered.

Theorem 6 Consider the perturbed sampled-data system of eqs. (1) and its discrete-time description (2)-(8). Express $A_{d}$ in Jordan canonical form as $A_{d}=V \Lambda V^{-1}$ and consider $V$ partitioned as in (17). Let the perturbation $\Delta y_{k}$ be bounded as in (18)-(19), where $z_{k}=V^{-1} x_{k}, \Theta_{p} \in \mathbb{R}_{+, 0}^{\mathrm{P} \times n}, \Theta_{c} \in$ $\mathbb{R}_{+, 0}^{\mathrm{M} \times n}, \theta_{p} \in \mathbb{R}_{+, 0}^{\mathrm{P}}, \theta_{c} \in \mathbb{R}_{+, 0}^{\mathrm{M}}$ and $\Theta_{s} \in \mathbb{R}_{+, 0}^{\mathrm{M} \times \mathrm{P}}$. Consider the matrix $M$ defined in (22)-(23) and suppose that $\rho(M)<1$. Then, given any $\epsilon \in \mathbb{R}_{+}^{n}$ and $x_{0} \in \mathbb{R}^{n}$, there exists $\ell=$ $\ell\left(\epsilon, x_{0}\right) \geq 0$ such that for all $t \geq t_{\ell}=\ell \mathrm{T}$,

$$
\begin{aligned}
\left|x_{p}(t)\right| & \leq \sup _{0 \leq \sigma<\mathrm{T}}\left[\bar{x}_{p}^{1}(\sigma)+\bar{x}_{p}^{2}(\sigma)+\bar{x}_{p}^{3}(\sigma)\right], \text { where } \\
\bar{x}_{p}^{1}(\sigma) & \triangleq\left|V_{p}+\Psi(\sigma)\left[A_{p} V_{p}+B_{p}\left(D_{c} C_{p} V_{p}+C_{c} V_{c}\right)\right]\right| \bar{z} \\
\bar{x}_{p}^{2}(\sigma) & \triangleq\left|\Psi(\sigma) B_{p} D_{c}\right| \bar{y}_{p}, \quad \bar{x}_{p}^{3}(\sigma) \triangleq\left|\Psi(\sigma) B_{p}\right| \bar{y}_{c}, \\
\bar{z} & \triangleq\left(b_{\infty}+\epsilon\right), \quad \bar{y}_{p} \triangleq \max \left\{\Theta_{p} \bar{z}, \theta_{p}\right\}, \\
\bar{y}_{c} & \triangleq \max \left\{\Theta_{c} \bar{z}+\Theta_{s} \bar{y}_{p}, \theta_{c}\right\},
\end{aligned}
$$

the supremum in (31) is taken componentwise, and $b_{\infty}$ is given by Theorem 2 .

PROOF. The proof follows straightforwardly from the solution of the system equations between sampling instants and the application of componentwise operations. See Haimovich $(2006, \S 6)$ for details.

\section{Examples}

To illustrate the application of the method developed, we consider the linearized model of the magnetic ball levitation system used in Ishii et al. (2004) and Ishii and Francis (2002, §4.7). The quantized sampled-data system consists of a continuous-time plant with matrices

$$
A=\left[\begin{array}{ccc}
0 & 1 & 0 \\
2798 & 0 & -19.6 \\
0 & 0 & -24.39
\end{array}\right], \quad B=\left[\begin{array}{c}
0 \\
0 \\
2.439
\end{array}\right]
$$

that is regularly sampled every $\mathrm{T}=4.605 \cdot 10^{-3}$ seconds. The state samples are multiplied by the feedback gain $K=\left[\begin{array}{lll}10315.67 & 195.02-49.47\end{array}\right]$ and then passed through a scalar quantizer $q$ to generate the control inputs at times $t_{k}=k \mathrm{~T}$, for all $k \geq 0$. At times $t_{k} \leq t<t_{k}+\mathrm{T}$, the plant input is held at its value by means of a zero-order hold device. The quantizer $q$ is defined in Ishii et al. (2004) and is of the semipractical logarithmic type. To apply the proposed ultimate bound estimation method to this system, we regard this system as a perturbed sampled-data system with describing equations (1), where the perturbations are introduced by the quantizer and bounded as was described in Section 2. To have a tight bound on the perturbation introduced by the scalar quantizer $q$ in Ishii et al. (2004), we write $q(s)=r \tilde{q}(s)$ and calculate the scalar $r$ so that $\Delta s=\tilde{q}(s)-s$ satisfies a tight bound of the form (10). Straightforward calculations yield $r=1.1289, \delta=0.2806$ and $u^{\circ}=0.5775$. We next put the system into the form (1), with the perturbation bounded as in (18)-(19). We thus have a continuous-time plant of equation (1a) with $A_{p}=A$, $B_{p}=B r$ and $C_{p}=\mathrm{I}_{3}$, and a static discrete-time controller of equation (1c) with $D_{c}=K$ and zero $C_{c}$. We also have (1d)-(1e), and note that $\mathrm{N}_{p}=3, \mathrm{~N}_{c}=0, \mathrm{M}=1, \mathrm{P}=3$, $n=\mathrm{N}_{p}+\mathrm{N}_{c}=3$ and $\mathrm{S}=\mathrm{P}+\mathrm{M}=4$. The discrete-time model (2)-(8) is then given by

$$
x_{k}=x_{p}\left(t_{k}\right) \quad A_{d}=A_{11} \quad B_{d}=\left[\begin{array}{ll}
B_{11} & B_{12}
\end{array}\right] .
$$

We express $A_{d}$ in Jordan canonical form as $A_{d}=V \Lambda V^{-1}$ and note that the partition of $V$ in (17) is just $V=V_{p} \in$ $\mathbb{C}^{3 \times 3}$, since $\mathrm{N}_{c}=0$. From (11) and (12), and since there is no quantization at the plant outputs, we have $\Gamma_{p}=\mathbf{0}_{3 \times 3}$ and $\theta_{p}=\mathbf{0}_{3 \times 1}$. Then, from (20), $\Theta_{p}=\mathbf{0}_{3 \times 3}$. From (11) and (12), we have $\Gamma_{c}=\delta$ and $\theta_{c}=u^{\circ} /(1+\delta)$. Also, from (20), $\Theta_{c}=\Gamma_{c}\left|K V_{p}\right|$. We compute the matrix $M$ in (22) and verify that $\rho(M)<1$. Then, using Theorem 2 , we have $\beta=$ $\left[\begin{array}{lll}0.1121 & 0.0336 & 0.0507\end{array}\right]^{\prime}$ and $\gamma=[6.0671 .8172 .745]^{\prime} \cdot 10^{-2}$. Iteration of the map $T$ defined in (25) from the initial condition $\beta$ yields (numerically) $b_{\infty}=\gamma=[6.0671 .8172 .745]^{\prime}$. $10^{-2}$. Application of Theorem 2 part 3 yields

$$
\limsup _{k \rightarrow \infty}\left|x_{p}\left(t_{k}\right)\right| \leq\left[\begin{array}{lll}
9.2 & 363.5 & 862.2
\end{array}\right]^{\prime} \cdot 10^{-4} \text {. }
$$

Moreover, from Theorem 6 using $\epsilon=\left[\begin{array}{lll}1 & 1 & 1\end{array}\right]^{\prime} \cdot 10^{-10}$, there exists $\ell=\ell\left(\epsilon, x_{0}\right) \geq 0$ such that for all $t \geq t_{\ell}=\ell \mathrm{T}$,

$$
\left|x_{p}(t)\right| \leq\left[\begin{array}{lll}
9.2 & 363.5 & 862.2
\end{array}\right]^{\prime} \cdot 10^{-4} .
$$

We observe some interesting features of this example. First, note that we have numerically obtained $b_{\infty}=\gamma$. Hence, the lower bound on the discrete-time componentwise ultimate bound provided by Theorem 2 is achieved. Second, note that the bound (39), that takes account of intersample behavior, is identical to the bound (38), which is only valid at the sampling instants. The equality of these bounds shows that, in this example, there is no conservativeness in the bounding procedure of Theorem 6 .

In Ishii et al. (2004), a randomized algorithm is developed that reduces conservatism in the analysis of sampled-data systems with quantizers. The approach in Ishii et al. (2004) can reduce conservatism not only in the ultimate bounds for a system, but also in the required sampling period. In addi- 
tion, a guaranteed decay rate to the ultimate bound is considered. Here, we are only interested in comparing the ultimate bound obtained in Ishii et al. (2004) with the componentwise bound (39). The ultimate bound obtained in Ishii et al. (2004) is, using our notation, $\left\|x_{p}(t)\right\| \leq 0.053$, for $t \geq t_{\ell^{\prime}}$. From (39), it follows that $\left\|x_{p}(t)\right\| \leq 0.0936$, for $t \geq t_{\ell}$. It is not surprising, perhaps, that the bound obtained in Ishii et al. (2004) is better than that provided by our method, since the algorithm in Ishii et al. (2004) involves the analysis of individual state trajectories. On the other hand, note that the componentwise bound (39) gives a tighter bound on the first two components of the state, which represent the position and velocity of the ball in the magnetic levitation system. In particular, the ultimate bound on the ball position, $9.2 \cdot 10^{-4}$, is more than 50 times lower than 0.053 .

To gain insight into how conservative our results may be, we have simulated the system from 200 initial conditions selected randomly from a uniform distribution in the cube $[-0.1,0.1]^{3} \in \mathbb{R}^{3}$. Our simulations show that after $1 \mathrm{sec}-$ ond of simulation time (more than 200 sampling instants), the state trajectories ultimately converged in all cases to the region $|x| \leq\left[\begin{array}{lll}1.3 & 4.4203 .9\end{array}\right]^{\prime} \cdot 10^{-4}$. Comparing this bound with (39), it appears that the bound (39) is still very conservative, especially the one corresponding to the second state component. However, we note that not many existing results can be directly and systematically applied to this example.

In an attempt to compare our results with other theoretical results (not numerical as that of Ishii et al., 2004), we intended to apply the method of Miller et al. (1988, 1989). For this specific example, that method requires that the system at the sampling instants be written as $x_{k+1}=A_{d} x_{k}+P\left(x_{k}\right)$, where a suitable norm of the perturbation term $P\left(x_{k}\right)$ must be bounded as an affine function of the corresponding norm of the state. It can easily be shown that if either the Euclidean or infinity norms are selected, then such result cannot provide any useful ultimate bound.

\section{Conclusions}

We have developed a novel systematic method to obtain componentwise ultimate bounds for perturbed sampled-data systems, especially when the perturbations arise due to the use of quantizers. This method is completely systematic, without requiring adjustment of parameters or selection of, e.g., appropriate probability density functions, norms or Lyapunov functions in order to provide an ultimate bound for a quantized sampled-data system. To the best of the authors' knowledge, this is the first time that a systematic method which can accommodate a setting as that depicted in Figure 1 with different quantizers in each signal has been proposed in the literature. We have illustrated the simplicity and potential of the method with a numerical example.

\section{References}

P. Antsaklis and J. Baillieul, eds. Special issue on networked control systems. IEEE Trans. Aut. Contr., 49(9), 2004.

J. E. Bertram. The effect of quantization in sampled-feedback systems. Trans. Amer. Inst. Elec. Engrs., 77, pt. 2:177-182, 1958.

N. Elia and S. K. Mitter. Stabilization of linear systems with limited information. IEEE Trans. Aut. Contr., 46(9):1384-1400, 2001.

B. D. Green and L. E. Turner. New limit cycle bounds for digital filters. Theoretical Computer Science, 35(4):365-374, 1988.

$\mathrm{H}$. Haimovich. Quantisation issues in feedback control. $\mathrm{PhD}$ thesis, School of Electrical Engineering and Computer Science, The University of Newcastle, Australia, March 2006.

R. A. Horn and C. R. Johnson. Matrix Analysis. Cambridge University Press, Cambridge, UK, 1985.

S. Huang, M.R. James, D. Nešić, and P.M. Dower. Analysis of input to state stability for discrete-time nonlinear systems via dynamic programming. Automatica, 41(12):2055-2065, 2005.

H. Ishii and T. Başar. Remote control of LTI systems over networks with state quantization. Systems and Control Letters, 54:15-31, 2005.

H. Ishii and B. A. Francis. Quadratic stabilization of sampled-data systems with quantization. Automatica, 39:1793-1800, 2003.

H. Ishii and B. A. Francis, editors. Limited data rate in control systems with networks. Lecture Notes in Control and Information Sciences 275. Springer-Verlag Berlin Heidelberg, 2002.

H. Ishii, T. Başar, and R. Tempo. Randomized algorithms for quadratic stability of quantized sampled-data systems. Automatica, 40:839-846, 2004.

Z. P. Jiang and Y. Wang. Input-to-state stability for discrete-time nonlinear systems. Automatica, 37(6):857-869, 2001.

H. Khalil. Nonlinear Systems. Prentice-Hall, New Jersey, 3rd edition, 2002.

E. Kofman. Non conservative ultimate bound estimation in LTI perturbed systems. Automatica, 41(10):1835-1838, 2005.

E. Kofman, H. Haimovich, and M. M. Seron. A systematic method to obtain ultimate bounds for perturbed systems. Int. Journal of Control, 80(2):167-178, 2007.

R. K. Miller, M. S. Mousa, and A. N. Michel. Quantization and overflow effects in digital implementations of linear dynamic controllers. IEEE Trans. Aut. Contr., 33(7):698-704, 1988.

R. K. Miller, A. N. Michel, and J. A. Farrell. Quantizer effects on steady-state error specifications of digital feedback control systems. IEEE Trans. Aut. Contr., 34(6):651-654, 1989.

D. Nešić, A. R. Teel, and E. D. Sontag. Formulas relating $\mathcal{K} \mathcal{L}$ stability estimates of discrete-time and sampled-data nonlinear systems. Systems and Control Letters, 38:49-60, 1999.

E. D. Sontag and Y. Wang. On characterizations of the input-tostate stability property. Systems and Control Letters, 24:351359,1995

D. Williamson. Digital control and implementation. Finite wordlength considerations. Prentice Hall, 1991.

W. S. Wong and R. W. Brockett. Systems with finite communication bandwidth constraints-II: stabilization with limited information feedback. IEEE Trans. Aut. Contr., 44(5):1049-1053, 1999.

S. Yakowitz and S. R. Parker. Computation of bounds for digital filter quantization errors. IEEE Trans. on Circuit Theory, CT-20 (4):391-396, 1973. 